\title{
Alcohol gifts in medicine
}

octors commonly receive gifts of alcohol in professional settings, particularly after giving a talk. This article reflects our personal opinions of this practice. We want our colleagues to consider the symbolism of giving or receiving alcohol as a gift in a professional setting. This is not a promotion of abstinence.

\section{“'Everyone drinks (alcohol)' is a frequently used phrase. This is not true as 20\% of Australians do not consume alcohol"}

Doctors should be aware of the preventable death and suffering caused by the harmful consumption of alcohol. Thirty per cent of emergency department attendances are alcohol related, ${ }^{1}$ and there are also personal health risks, mental health issues and social issues involved. Balanced against this, the health benefits of drinking alcohol are not clearly established. ${ }^{2}$ There is little to be said in favour of the promotion of alcohol, but consumption is a personal choice. In contrast, gift giving of alcohol in professional settings is a public statement.

Many professional bodies have policies on alcohol consumption, but we could find none - including the websites of the Royal Australasian College of Physicians, Royal Australasian College of Surgeons, and Royal Australian College of General Practitioners - with policies on giving alcohol as gifts. Health care professionals have a duty to promote health; as individuals and in our professional groups. The provision of alcohol as a gift would seem to be contrary to a basic tenet of medical practice, which is to promote health.

Alcohol consumption is an accepted part of Australian culture. Our impression is that no one has questioned the appropriateness of

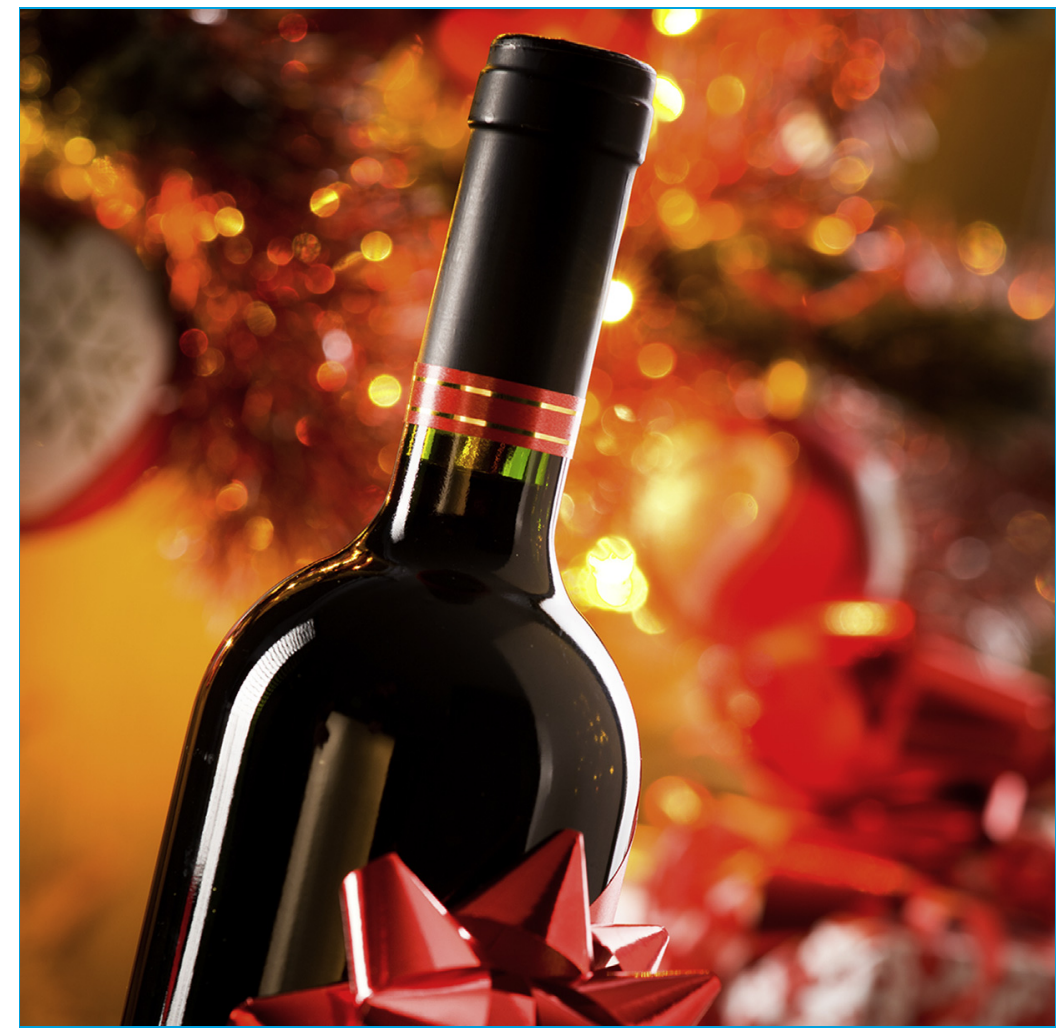

alcohol as a gift in the health professional setting. "Everyone drinks (alcohol)" is a frequently used phrase. This is not true as $20 \%$ of Australians do not consume alcohol. ${ }^{3}$ Therefore, the recipient of the alcohol gift could be pregnant, a recovering alcoholic or from a cultural or religious tradition that prohibits alcohol consumption. Others just do not consume alcohol. Giving alcohol as a gift in a professional setting is an ingrained habit, with little thought given to the choice of gift, consideration of the recipient or symbolic consequences of the act.

There are alternatives to giving alcohol as a gift. Giving a gift to someone who has taken the time to prepare and give a talk cannot compensate him or her adequately for his or her time, but it is a token of appreciation. So some thought on the part of the gift giver is in order. International speakers could have something from the country they are visiting and, similarly, someone from interstate could receive something particular to that state. If something particular cannot be identified, general gifts might include chocolates or flowers. Book or music vouchers are always valued. There are also charities that provide online gifts.

In summary, gift giving of alcohol in a professional setting is inappropriate in that it may be insensitive to the recipient and is a poor health promotion message. We call for professional health bodies to take a leadership role and develop policies to find appropriate alternatives to giving alcohol as a gift. We challenge health professionals to be more thoughtful in their choice of gifts in a professional setting.

Competing interests: No relevant disclosures.

(c) 2015 AMPCo Pty Ltd. Produced with Elsevier B.V. All rights reserved.

References are available online at www.mja.com.au. 
1 Egerton-Warburton D, Gosbell A, Wadsworth A, et al. Survey of alcohol-related presentations to Australasian emergency departments. Med J Aust 2014; 201: 584-587. https://www.mja. com.au/journal/2014/201/10/survey-alcohol-relatedpresentations-australasian-emergency-departments

2 Knott CS, Coombs N, Stamatakis E, et al. All cause mortality and the case for age specific alcohol consumption guidelines: pooled analyses of up to 10 population based cohorts. BMJ 2015; 350: h384.

3 Australian Institute of Health and Welfare. National Drug Strategy Household Survey detailed report 2013. Canberra: AlHW. (AlHW Cat No. PHE 183; Drug Statistics Series No. 28.) www.aihw.gov.au/publication-detail/?id=60129549469 (accessed Oct 2015). 